\title{
Parathyroid Hormone in Human Plasma
}

\author{
IMMUNOCHEMICAL CHARACTERIZATION AND \\ BIOLOGICAL IMPLICATIONS
}

\author{
Gino V. Segre, Joel F. Habener, David Powell, Geoffrey W. Tregear, and \\ John T. PotTs, JR.
}

From the Endocrine Unit, Department of Medicine, Massachusetts General

Hospital and Harvard Medical School, Boston, Massachusetts 02114

A B S T R A C T Antigenic recognition of four anti-bovine parathyroid hormone antisera was characterized by their reactivity with bovine hormonal fragments (1-34, $1-13,14-34,19-34,53-84)$ and human hormone extracted from parathyroid adenomas. All antisera were found to have antibody populations which recognized more than one antigenic determinant and all antisera differed in their specificity and reactivity for the fragments of bovine hormone. By modification of two antisera, GP-1 and GP-133, by preincubation with excess concentrations of 1-34 or 53-84 fragments, antigenic recognition was restricted to defined regions of the hormonal sequence.

When assays using these modified antisera were applied to the study of hormones extracted from glands, greater immunochemical similarities were seen between bovine and human parathyroid hormone using assays that were specific for the measurement of amino-terminal portions of the hormones than of the carboxy-terminal portions.

When assays using these antisera were applied to the study of endogenous parathyroid hormone in human plasma, the immunoreactive hormone in the general circulation was shown to substantially lack an amino-terminal portion of the sequence of the intact hormone, including an antigenic determinant requiring all or some of the 14-19 region. This deletion accounts, at least in part, for the immunochemical heterogeneity of plasma

This work has been presented in part at the Annual Meetings of the American Society for Clinical Investigation and American Federation for Clinical Research, 30 April 1972.

Dr. Habener is a Special Fellow of the National Institute of Arthritis and Metabolic Diseases of the National Institutes of Health.

Received for publication 5 May 1972 and in revised form 1.5 August 1972. parathyroid hormone in man. Radioimmunoassay of fractions of peripheral plasma subjected to gel filtration confirms that the dominant form of the immunoreactive hormone in the general circulation of man is a hormonal fragment that is totally devoid of amino-terminal reactivity. Because of this deletion, it can be concluded that most of the immunoreactive parathyroid hormone in the general circulation of man must be biologically inactive.

\section{INTRODUCTION}

In studies employing several antisera to bovine parathyroid hormone (BPTH), Berson and Yalow (1) reported that, with one of these antisera, the slope of inhibition of tracer binding by successively increasing aliquots of immunoreactive parathyroid hormone (PTH) in the plasma of patients with hyperparathyroidism differed from that seen with hormone extracted from human parathyroid adenomas. This indicated that $\mathrm{PTH}$ in man was immunochemically heterogeneous and that certain antisera recognized antigenic differences, presumably reflecting structural differences, between the plasma and stored hormone. A preliminary report by Canterbury and Reiss (2) indicated that several immunoreactive fractions could be detected after gel filtration of circulating PTH in man and studies in this laboratory (3) have shown that PTH in the venous effluent both of human parathyroid adenomas and normal bovine parathyroid glands is indistinguishable from hormone extracted from glands, but that $\mathrm{PTH}$ in the general peripheral circulation consists largely of a hormonal fragment which is immunochemically distinct from hormone extracted

\footnotetext{
${ }^{1}$ Abbreviations used in this paper: BPTH, bovine parathyroid hormone; HPTH, human parathyroid hormone; PTH, parathyroid hormone.
} 
from glands and has an estimated molecular weight that is 2000 daltons less than that of intact hormone. These latter results, in vivo, indicated that cleavage of the stored form of the hormone occurred after release from the gland and not at the time of release as had been suggested by earlier organ culture studies done in vitro (4, $5)$. More recent studies, by Martin, Greenberg, and Melick (6), using human parathyroid monolayer cell cultures, have shown that the secreted hormone is indistinguishable from the hormone extracted from human adenomas by gel filtration and by polyacrylamide gel electrophoresis. These results are consistent with the findings observed in vivo (3).

To further evaluate the immunochemical properties of endogenous, circulating $\mathrm{PTH}$ in man, it was necessary to develop radioimmunoassays using antisera that react specifically with defined regions of the hormone. The recent determination of the complete amino acid sequence of $\operatorname{BPTH}(7,8)$ and the availability of both natural (7) and synthetic hormonal fragments (9) of $\mathrm{BPTH}$ have permitted us to characterize the antigenic recognition of several antisera. Antibody populations within these antisera are then selectively blocked by preincubation with excess concentrations of the appropriate fragments of the $\mathrm{BPTH}$ sequence. Antigenic recognition is thereby restricted to specific regions of the hormonal molecule. Use of these modified antisera enabled us to develop radioimmunoassays that measure specific regions of the hormone and apply them to a study of the immunochemical nature of endogenous PTH in man. The results from these studies have allowed us to further understand the biological significance of PTH metabolism. In addition, these results have important implications for the use of the radioimmunoassay for measuring $\mathrm{PTH}$ in the circulation of man.

\section{METHODS}

Preparation of hormones and hormonal fragments. Purified BPTH (10) and a partiolly nurified preparation of human parathyroid hormone $(\mathrm{HPTH})(11,12)$ were used as standards in the radioimmunoassay. Aliquots of BPTH were iodinated with ${ }^{125} \mathrm{I}$ by a modification of the method of Hunter and Greenwood (13) for use as tracer $(300 \mathrm{Ci} / \mathrm{g})$.

Bovine hormonal fragments 1-34, 14-34, 19-34, and 1-13 were synthesized by a modification of the solid phase methods previously described (9). Fragments 1-34, 14-34, and 19-34 were prepared by removing quantities of peptide-resin at the appropriate steps in a single synthesis beginning with resin-esterified phenylalanine. Using the test systems previously described (9), this preparation of fragment $1-34$ was found to be more active than the initial preparation both in vivo and in vitro. In eight separate bioassays, based on the activation of rat renal cortical adenyl cyclase (14), the potency of the more recently synthesized preparation ranged from 1800 to 2300 USP $\mathrm{u} / \mathrm{mg}$. Fragment $1-13$ was also prepared by a modification of the solid phase methods beginning with resin-esterified lysine.
The hormonal fragment 53-84 was prepared by tryptic digestion of native hormone after blockade of lysine $\epsilon$-amino groups with maleic anhydride as previously described (7).

Amino acid composition and precise quantitation of preparations of purified $\mathrm{BPTH}$, synthetic and natural hormonal fragments of $\mathrm{BPTH}$, and partially purified $\mathrm{HPTH}$ werc determined by combined acid and enzymatic hydrolysis techniques $(7,10)$.

Antisera. Antisera from guinea pigs that had been immunized with partially purified BPTH (10) were used at a final dilution of $1 / 300,000$ (GP-1), 1/80,000 (GP-118). $1 / 6000$ (GP-133), and $1 / 3000$ (GP-144), chosen to give a bound to free ratio of radioiodinated hormone in the absence of added hormone (antibody control) of 0.7-1.0.

Antigenic recognition of the antisera was restricted by preincubation of GP-1 and GP-133 (dilution of $1 / 30,000$ and $1 / 600$, respectively), $6-18 \mathrm{hr}$ before use in the radioimmunoassay with amounts of fragments $1-34$ or 53-84 calculated to give a final concentration of 20 and $10 \mathrm{ng}$, respectively, per assay tube. To insure that preincubation with a given fragment totally eliminated antibody recognition for that region of the sequence, binding of the antibody control was compared with identically prepared tubes to which an additional $10 \mathrm{ng}$ of the fragment used for preincubation was added. In no instance was tracer binding further inhibited. Preincubated antisera were used at final concentrations of $1 / 150,000$ (GP-1) and 1/3000 (GP-133).

Radioimmunoassay's. Radioimmunoassays were carried out by a modification (15) of the method of Berson, Yalow, Aurbach, and Potts (16), using 5,000-10,000 cpm of BPTH${ }^{125} \mathrm{I}, 100 \mu \mathrm{l}$ of one of the four antisera, and plasma samples or hormonal preparation adjusted to a final volume of $0.5 \mathrm{ml}$ with $0.05 \mathrm{M}$ veronal buffer-15\% v/v human plasma. Nonequilibrium incubation conditions $(17,18)$ for $6-7$ days at $4^{\circ} \mathrm{C}$ and a modification of the charcoal-dextran phase separation (19) were used. In this modification, $200 \mathrm{ml}$ of cold $\left(4^{\circ} \mathrm{C}\right) 0.05$ 2. veronal buffer was added to each of two beakers, one containing $10.0 \mathrm{~g}$ of Norit A charcoal (Fisher Scientific Co., Pittsburgh, Pa.) and the other containing $1.0 \mathrm{~g}$ of dextran T80 (Pharmacia, Uppsalla, Sweden). After the dextran was in solution and an homogeneous suspension of the charcoal achieved ( $\frac{1}{2} \mathrm{hr}$ of continuous mixing), the contents of both beakers were combined and mixed for $2 \mathrm{hr}$ at $4^{\circ} \mathrm{C}$. While the suspension was continuously stirring, $200-\mu 1$ aliquots were removed and added to the assay tubes $\left(10 \times 75 \mathrm{~mm}\right.$ flint glass). After $30-60 \mathrm{~min}$ at $4^{\circ} \mathrm{C}$, the tubes were then mixed on a Vortex mixer (Scientific Industries, Inc., Queens Village, N. Y.) and centrifuged for $10 \mathrm{~min}$ at $2000 \mathrm{rpm}$ in an International refrigerated centrifuge (model PR-2). The supernates were then carefully decanted into clean test tubes. ${ }^{125} \mathrm{I}$ in both the bound and free fractions was counted in a gamma well-spectrometer (Packard Instrument Co., Inc., Downers Grove, Ill.). PTH concentrations in all plasma samples were measured in multiple dilutions and all samples were assayed at least in duplicate. often in quadruplicate. Appropriate controls of samples without added antisera were used in each assay and for each sample to correct for nonspecific effects due to incubation damage of radioiodinated hormone $(20)$.

Gel filtration. Portions of $0.1-0.8 \mathrm{ml}$ of plasma and marker (BPTH- ${ }^{125} \mathrm{I}-20,000 \mathrm{cpm}, 40 \mathrm{pg}$ ) were chromato-

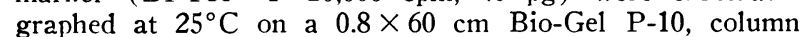
(100-200 mesh, Bio-Rad Laboratories, Richmond, Calif.) at a flow rate of approximately $8 \mathrm{ml} / \mathrm{hr}$, using an eluting buffer of $0.05 \mathrm{M}$ veronal-0.01 M disodium ethylenediamine- 

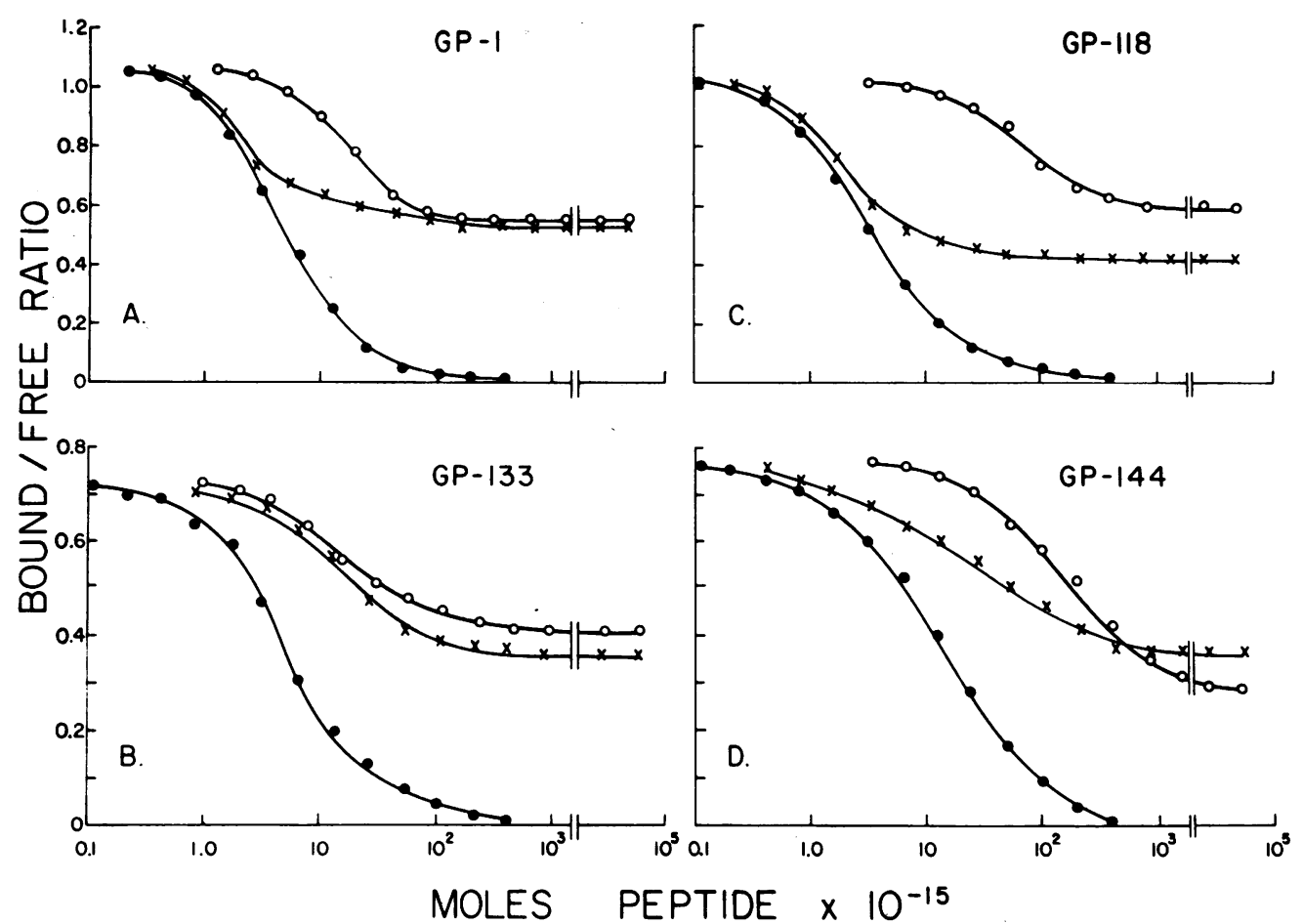

FIGURE 1 Comparison of the fall of antibody-bound to free BPTH- ${ }^{125} \mathrm{I}$ tracer from antibody control (bound to free ratio in the absence of unlabeled hormone) in assays using four antisera as a function of increasing concentrations of BPTH and BPTH fragments 1-34 and 53-84. Concentration is expressed in moles $\times 10^{-15}$. A. GP-1 $(1 / 300,000)$; B. GP-133 $(1 / 6000)$; C. GP-118 (1/80,000); D. GP-144 (1/3,000);

tetraacetate- $0.05 \%$ Merthiolate-10\% (v/v) human plasma by methods previously described (3).

Plasma samples. Venous blood was collected in heparinized tubes from the antecubital vein from 24 patients in the fasting state. 19 had hyperparathyroidism and 5 were normal subjects (21). Samples from veins draining the parathyroid glands were collected at the time of diagnostic transfemoral catheterization in five patients with surgically proven parathyroid adenomas and in two patients without disorders of parathyroid function (cases 23 and 27 in reference 22). The latter blood samples contain hormonal concentrations that were as much as 50 -fold greater than were found in the general peripheral circulation (22).

\section{RESULTS}

Characterization of antigenic recognition of antiBPTH antisera. Fig. 1 compares the inhibition of binding of BPTH- ${ }^{120}$ I tracer to four antisera by BPTH and fragments 1-34 and 53-84. The partial inhibition of tracer binding by both fragments with all four antisera shows that all the antisera contain antibodies of high affinity to antigenic determinants within the selected sequences. The concentration of each fragment required for inhibition of tracer binding differs for each antiserum, as does the per cent of total inhibition observed with addition even in marked excess of each fragment. The carboxy-terminal fragment inhibits tracer binding more effectively with all antisera, but where the slopes of inhibition of tracer binding by fragments are sufficiently alike to permit comparison of the concentration of fragments required for equivalent inhibition of tracer binding the ratio of concentration of fragments 1-34 to 53-84 varies from $1.2 / 1$ for GP-133 to $50 / 1$ for GP-118 (Fig. $1 \mathrm{~B}, \mathrm{C}$ ).

When PTH in the general circulation is measured in assays using GP-133, the slopes of inhibition of tracer binding by successively increasing aliquots of sample are identical with that caused by HPTH, but when GP-1 is used there is evident nonidentity of these two slopes (see below). Therefore, these antisera were chosen for further characterization.

Fig. 2 shows the inhibition of binding of $\mathrm{BPTH}-{ }^{125} \mathrm{I}$ to each antiserum by fragment $1-34$ and subfragments $1-13,14-34$, and 19-34 after each antiserum was preincu- 


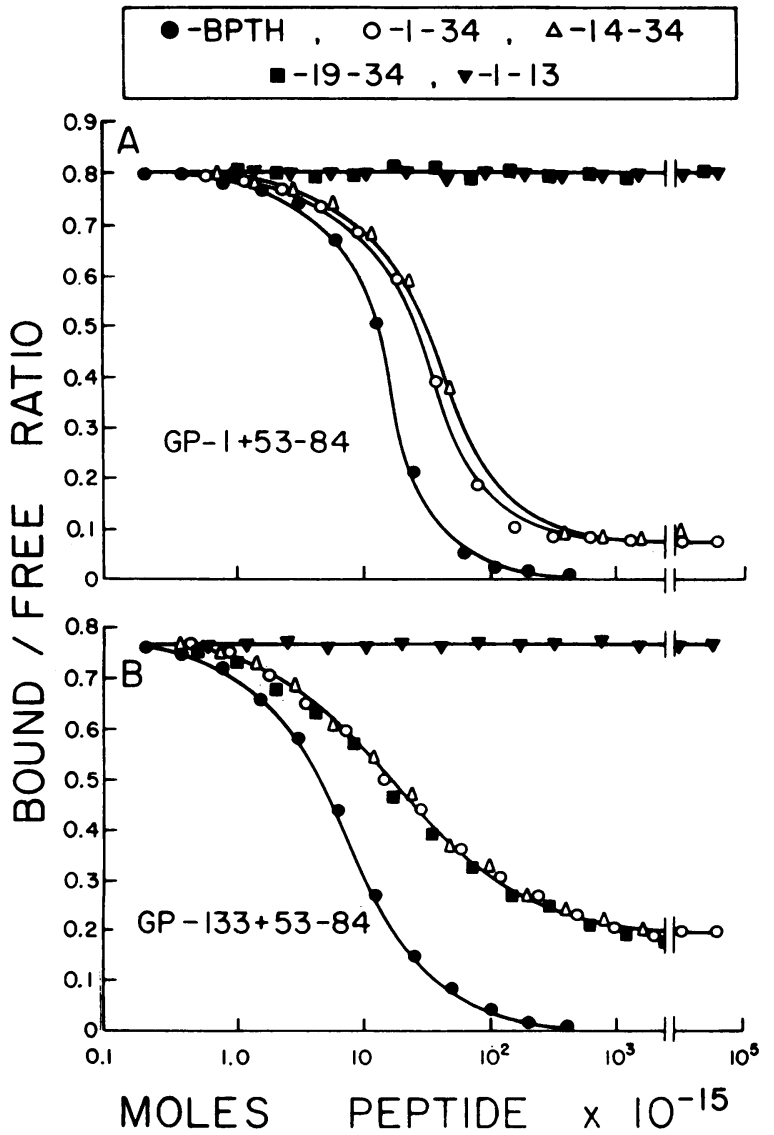

Figure 2 Comparison of the inhibition of binding of $\mathrm{BPTH}$ ${ }^{125} \mathrm{I}$ tracer by increasing concentrations of $\mathrm{BPTH}$, fragment $1-34$, and subfragments $1-13,14-34$ and $19-34$ from A. GP-1 $(1 / 150,000)$ preincubated with excess $53-84$; B. GP-133 $(1 / 3,000)$ preincubated with excess $53-84$. Concentration is expressed in moles $\times 10^{-15}$.

bated with excess fragment 53-84. In assays with GP-1 equimolar concentrations of 1-34 and 14-34 give identical curves of inhibition of tracer binding, whereas subfragments 1-13 and 19-34 do not inhibit tracer binding in concentrations as high as $6 \times 10^{-11} \mathrm{M}$ and $5 \times 10^{-11} \mathrm{M}$, respectively. This demonstrates that the major antigenic determinant within the amino-terminal 34 residues for GP-1 requires all or some of the 14-19 region of the molecule (Fig. 2A).

In assays using GP-133, equimolar concentrations of fragments 1-34 and subfragments 14-34 and 19-34 give identical curves of inhibition of tracer hinding. thus demonstrating that a major antigenic determinant(s) within the amino-terminal 34 residues for GP-133 must be within the 19-34 sequence (Fig. 2B). Subfragment 1-13 fails to inhibit tracer binding in concentrations as high as $6 \times 10^{-11} \mathrm{M}$. These findings indicate that the first 18 residues, at least, are of no immunological importance for GP-133. Of course, it cannot be excluded that the region of recognition may include a portion of the sequence which is carboxy-terminal to position 34 . Therefore, a longer fragment which is extended to include this region might inhibit binding of $\mathrm{BPTH}_{-}{ }^{125} \mathrm{I}$ tracer more effectively.

In addition, when saturating concentrations of fragments 1-34 and 53-84 are added together, they inhibit $95 \%$ of binding of BPTH- $-{ }^{125} \mathrm{I}$ to GP-1, but only $85 \%$ of tracer binding to GP-133. This indicates that antigenic determinant(s) are present within the 35-52 region which are recognized by both antisera. Since neither fragment 1-34 or its subfragments can compete with $\mathrm{BPTH}_{-}{ }^{125} \mathrm{I}$ for this additional antigenic recognition site(s), this probably explains the more effective inhibition of tracer binding by BPTH than by the hormonal fragments seen in Fig. 2 (1-34 and 14-34 in the case of GP-1 and 1-34, 14-34 and 19-34 in the case of GP-133).

Studies of BPTH and HPTH using GP-1 and GP-13.3 and each antiserum after preincubation. Fig. $3 \mathrm{com}-$ pares the relative curves of inhibition of binding of BPTH $-{ }^{125}$ I tracer by BPTH and HPTH in assays using GP-1 and GP-133 and each antiserum after preincubation with excess $1-34$ or $53-84$ fragment. With all six assay systems, detection for $\mathrm{BPTH}$ is better than for HPTH. In assays with both antisera, before preincubation (Fig. 3A,D), four to five times more HPTH than $\mathrm{BPTH}$ is required to produce a $50 \%$ depression of the bound to free ratio. However, the reactivity of $\mathrm{HPTH}$ relative to BPTH is increased by 2 - to $2 \frac{1}{2}$-fold in assays using GP-1 and GP-133 after preincubation with excess concentrations of 53-84 (Fig. 3B,E). In contrast, in assays using GP-1 preincubated with excess concentrations of 1-34 fragment, eight times more HPTH than BPTH is required for $50 \%$ depression of the bound to free ratio and in assays using GP-133 preincubated with excess $1-34$, the slopes of inhibition of tracer binding by the two hormones are no longer identical (Fig. 3C,F). Although BPTH and HPTH clearly differ immunochemically, the lower ratio of cross-reactivity of HPTH to BPTH observed in assays using both antisera preincubated with excess 53-84 indicates that greater immunochemical similarities exist in the amino portion of the sequence.

Radioimmunoassay of endogenous PTH in man. Unblocked GP-1 and GP-133 and the 1-34 and 53-84 preincubated modifications of both antisera were used, simultaneously, to assay PTH in parathyroid effluent and peripheral plasma from normal subjects and from patients with hyperparathyroidism. Fig. 4 shows that the slope of inhibition of tracer binding caused by increasing aliquots of PTH from the parathyroid effluent plasma of one of these patients with hyperparathyroidism $(\mathrm{T} \mathfrak{u})$ is identical with that caused by HPTH. The concentration of immunoreactive material as measured in all six systems is virtually identical (range $18-25 \mathrm{ng} / \mathrm{ml}$ ) indicating that 

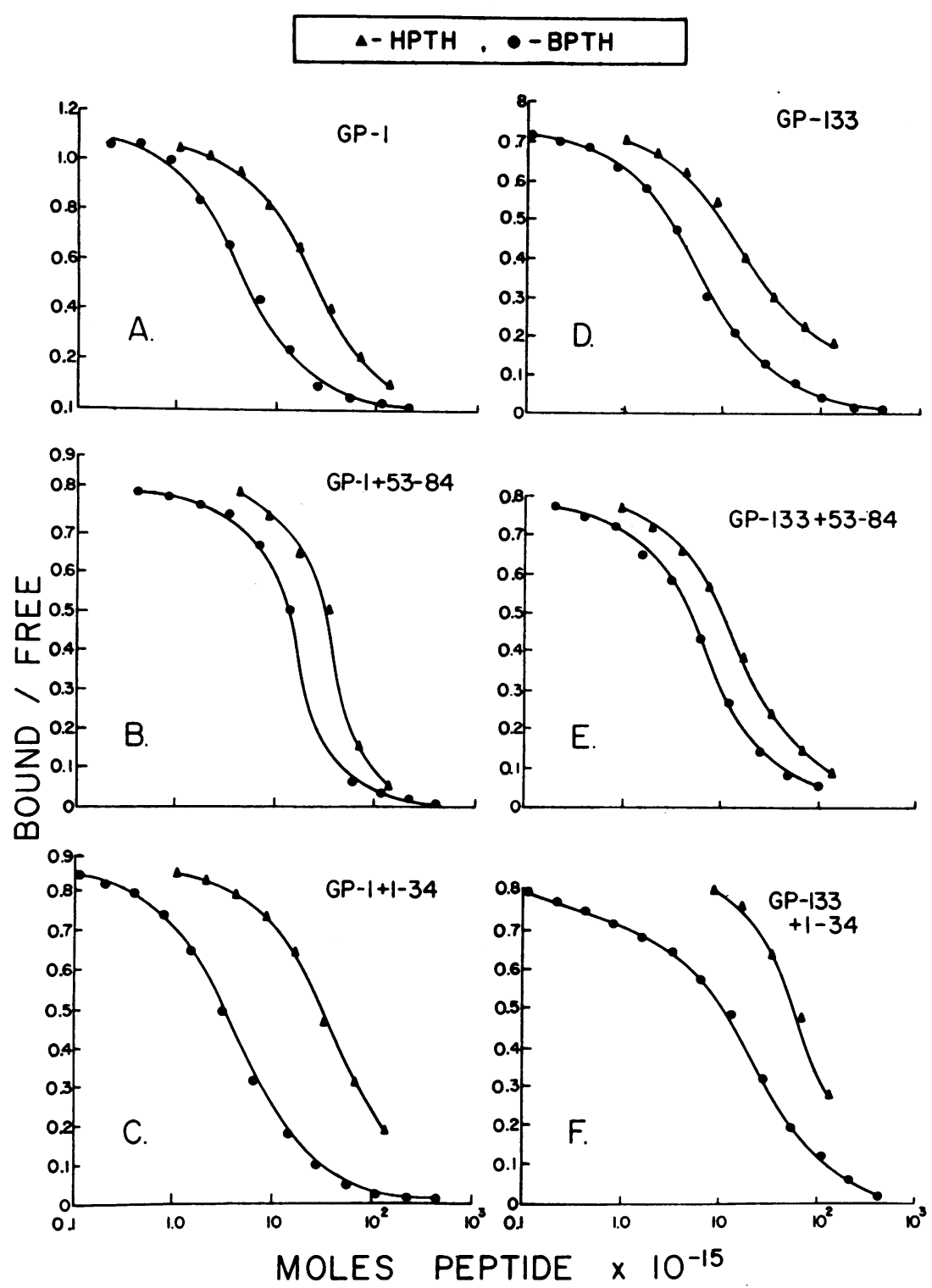

FIGURE 3 Comparison of the inhibition of binding of BPTH- ${ }^{105} \mathrm{I}$ tracer by increasing concentrations of BPTH and HPTH, from A. GP-1 $(1 / 300,000)$; B. GP-1 $(1 / 150,000)$ preincubated with excess $53-84$; C. GP-1 $(1 / 150,000)$ preincubated with excess $1-34$; D. GP-133 $(1 / 6,000)$; E. GP-133 (1/3,000) preincubated with excess 53-84; F. GP-133 (1/3,000 preincubated with excess 1-34. Concentration is expressed in moles $\times 10^{-15}$.

all antigenic determinants recognized by these antisera are present in equal concentrations. When PTH samples from the general circulation of this patient are assayed using GP-133 and its two preincubated modifications, the slope of inhibition of tracer binding by increasing aliquots of sample is again identical with that caused by HPTH (Fig. 4D, E, F). In contrast, in assays using GP-1, the two slopes of inhibition of tracer binding are clearly different (Fig. 4A). However, in assays using GP-1 preincubated with either excess $1-34$ or $53-84$ fragments, the two slopes become essentially identical (Fig. 4B,C). The identical slopes of inhibition of tracer binding given by samples from the general circulation and HPTH can be better appreciated when peripheral plasma from patients; $\mathrm{Me}$ and Sh (Table I) with higher concentrations of immunoreactivity are examined (Fig. $5)$. Over a wide range of depression of the bound to free ratio (over $90 \%$ depression in assays using GP-1 preincubated with excess $1-34$ (Fig. 5A) and over $70 \%$ in assays using GP-1 preincubated with excess 53-84 


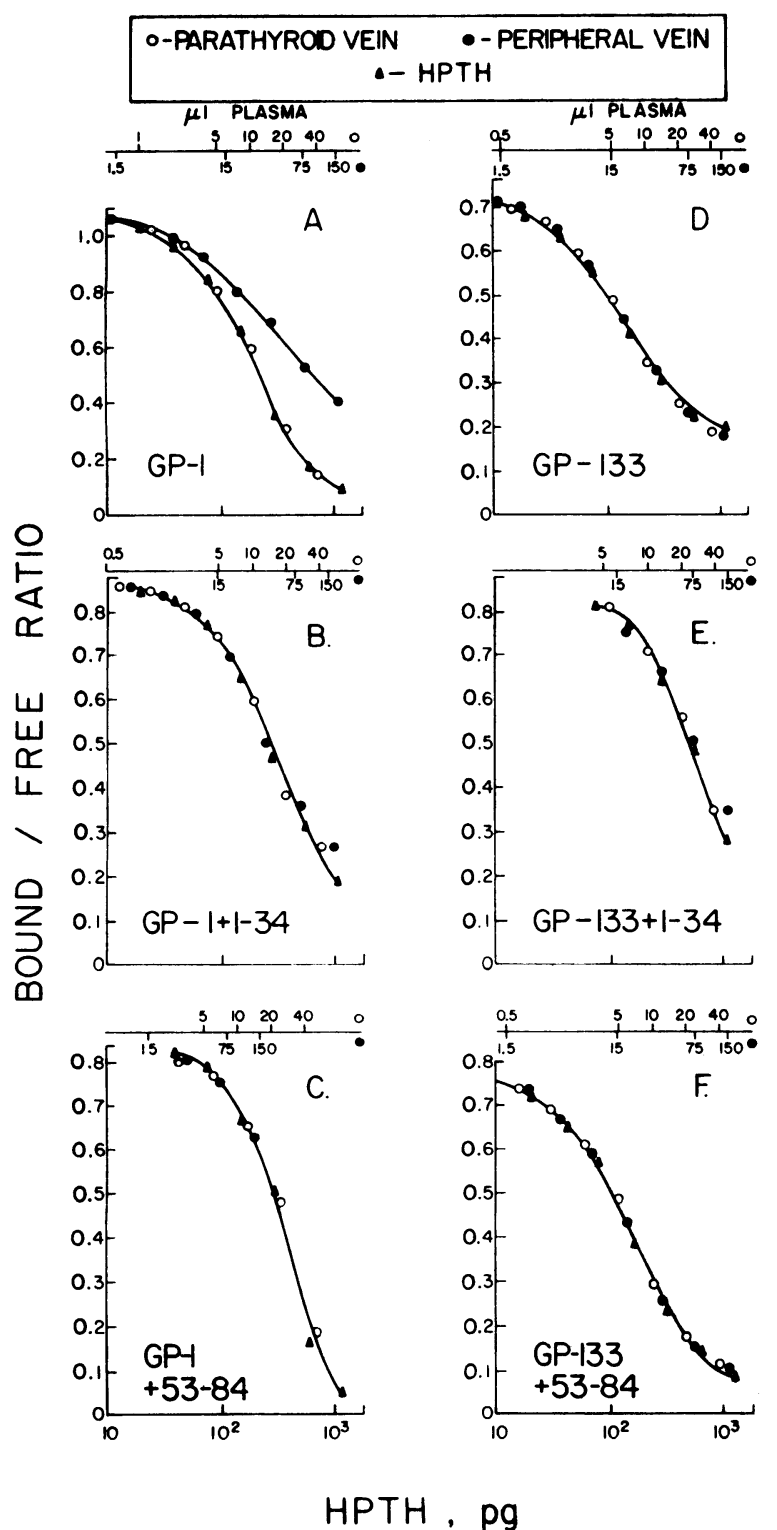

FIGURE 4 Comparison of the immunoreactive PTH in serially diluted plasma samples from parathyroid effluent and peripheral veins from patient, $\mathrm{Tu}$, in assays using, A. GP-1 $(1 / 300,000) ;$ B. GP-1 $(1 / 150,000)$ preincubated with excess 1-34; C. GP-1 (1/150,000) preincubated with excess 53-84; D. GP-133 $(1 / 6,000)$; E. GP-133 $(1 / 3,000)$ preincubated with excess $1-34$; F. GP-133 $(1 / 3,000)$ preincubated with excess 53-84. Concentration is expressed in picograms of HPTH.

(Fig. 5B), samples from both patients give slopes of inhibition of tracer binding which are identical with that given by HPTH. Therefore, accurate quantitation of the immunoreactivity in peripheral plasma samples is possible.

In the peripheral plasma from patient, $\mathrm{Tu}$, the concentration of immunoreactive material measured in as- says with GP-1 preincubated with excess 1-34 fragment is identical with that measured using GP-133 and its preincubated modifications (range 7.7-8.5 ng/ml), (Fig. 4B, D, E. F). However, the concentration measured in assays with GP-1 preincubated with excess 53-84 fragment is only $1.4 \mathrm{ng} / \mathrm{ml}$, or one-sixth as much (Fig. $4 \mathrm{C}$ ).

TABLE I

Immunoreactive PTH in Plasma Measured by GP-I Alternately Preincubated with Excess 1-34 and 53-84 Fragments

\begin{tabular}{lll}
$\begin{array}{c}\text { Patient and } \\
\text { plasma source }\end{array}$ & Gormone concentration* & $\begin{array}{c}\text { Ratio } \\
\text { GP-1 }+1-34||\end{array}$ \\
\cline { 2 - 3 } & $n g / m l$ & GP-1 + 53-84
\end{tabular}

I. Parathyroid vein

A. Patients with hyperparathyroidism (5)

$\begin{array}{lrrr}\mathrm{Cl} & 8.1 & 7.2 & 1 \\ \mathrm{De} & 6.0 & 5.1 & 1 \\ \mathrm{Si} & 20.0 & 20.0 & 1 \\ \mathrm{Tu} & 25.0 & 18.0 & 1 \\ \mathrm{Ve} & 40.0 & 40.0 & 1\end{array}$

B. Patients with normal parathyroid function (2)

$\begin{array}{llll}\mathrm{Fe} \uparrow & 10.0 & 10.6 & 1 \\ \mathrm{Ha} \uparrow & 12.0 & 11.0 & 1\end{array}$

II. Antecubital vein

A. Patients with hyperparathyroidism (19)

$\begin{array}{lrrr}\mathrm{Al} & 20.0 & 1.8 & 11 \\ \mathrm{Bc} & 15.3 & 2.1 & 7 \\ \mathrm{Br} & 4.0 & 0.3 & 13 \\ \mathrm{Co} & 22.0 & 2.0 & 11 \\ \mathrm{Gr} & 6.7 & 1.0 & 7 \\ \mathrm{Gro} & 7.2 & 1.8 & 4 \\ \mathrm{Hi} & 8.4 & 2.3 & 4 \\ \mathrm{Mc} & 16.4 & 2.3 & 7 \\ \mathrm{Me} & 11.1 & 3.0 & 4 \\ \mathrm{Pe} & 4.0 & 0.2 & 20 \\ \mathrm{Ry} & 14.5 & 2.0 & 7 \\ \text { San } & 19.5 & 2.6 & 7 \\ \text { Sh } & 12.1 & 3.1 & 4 \\ \text { Tu } & 8.1 & 1.4 & 6 \\ \text { Tuc } & 12.4 & 2.6 & 5 \\ \text { Tun } & 25.0 & 2.5 & 10 \\ \text { Va } & 6.7 & 1.1 & 6 \\ \text { Ve } & 15.8 & 1.2 & 8 \\ \text { IVo } & 19.6 & 2.5 & \end{array}$

B. Patients with normal parathyroid function** (5)

$$
\begin{array}{lll}
\text { Range } & 0.5-0.8 & \text { N.D. }
\end{array}
$$

* Mean of 4-10 assay determinations.

‡ GP-1 preincubated with excess fragment 1-34.

\$ GP-1 preincubated with excess fragment 53-84.

II Ratio of mean values expressed to nearest integer.

$\tau \mathrm{Fe}$ is case 23 and $\mathrm{Ha}$ is case 27 in reference 22 .

** Normal subjects in reference 21 .

$\ddagger \ddagger$ N.D. $=$ not detectable. 
Six additional parathyroid effluent samples and 23 peripheral venous samples were assayed using GP-1 alternately preincubated with excess 1-34 and 53-84 fragments (Table I). Four parathyroid effluent samples were from patients with hyperparathyroidism and two were from patients who were free of disorders of parathyroid function. 18 peripheral venous samples were from patients with hyperparathyroidism and five were from normal subjects.

PTH in the parathyroid effluent plasma from all patients has equivalent concentrations of immunoreactive material in both assays. However, in all 23 peripheral venous samples higher concentrations are found in assays using GP-1 preincubated with excess 1-34 than in assays using GP-1 preincubated with excess 53-84. In the samples from the patients with hyperparathyroidism, the concentration of immunoreactive material measured in the two assays differs from 4 - to 20 -fold. In assays using GP-1 preincubated with excess 1-34, the samples from the five normal subjects give a concentration range of 0.5 $0.8 \mathrm{ng} / \mathrm{ml}$. However, even though assays using GP-1 preincubated with excess 53-84 are of sufficient sensitivity to easily detect $0.5 \mathrm{ng} / \mathrm{ml}$, none of the samples were measurable. Thus, the observations made from examination of peripheral venous samples from patients with hyperparathyroidism are confirmed, qualitatively, by analysis of peripheral venous plasma from normal subjects.

These observations are further clarified when parathyroid effluent and peripheral venous plasma samples are subjected to gel filtration and each fraction assayed with these two preincubated modifications of GP-1. Parathyroid effluent samples from 7 patients and peripheral venous samples from 10 patients were studied. Five parathyroid-effluent and all peripheral-venous plasmas were from patients with hyperparathyroidism. Two parathyroid-effluent samples were from patients who were free of disorders of parathyroid function. Representative elution patterns are shown in Fig. 6. In all patients, regardless of their parathyroid status, PTH in the parathyroid effluent plasma elutes as a single peak, coincident with intact hormone marker, and contains equivalent concentrations of immunoreactive material in both assays (Fig. 6A). In contrast, most of the immunoreactive hormone in the general peripheral circulation sample elutes later than the BPTH marker and is measured only in assays using GP-1 preincubated with excess 1-34 (Fig. $6 \mathrm{~B})$. The peak of immunoreactivity measured in assays using GP-1 preincubated with $53-84$ is much smaller, and is coincident with the peak of intact hormone. Preincubation of the plasma sample in $8 \mathrm{~m}$ urea before gel filtration did not alter either the elution characteristics or the immunoreactivity of the hormone in the sample. Thus, it is quite unlikely that conformational change of the hor-

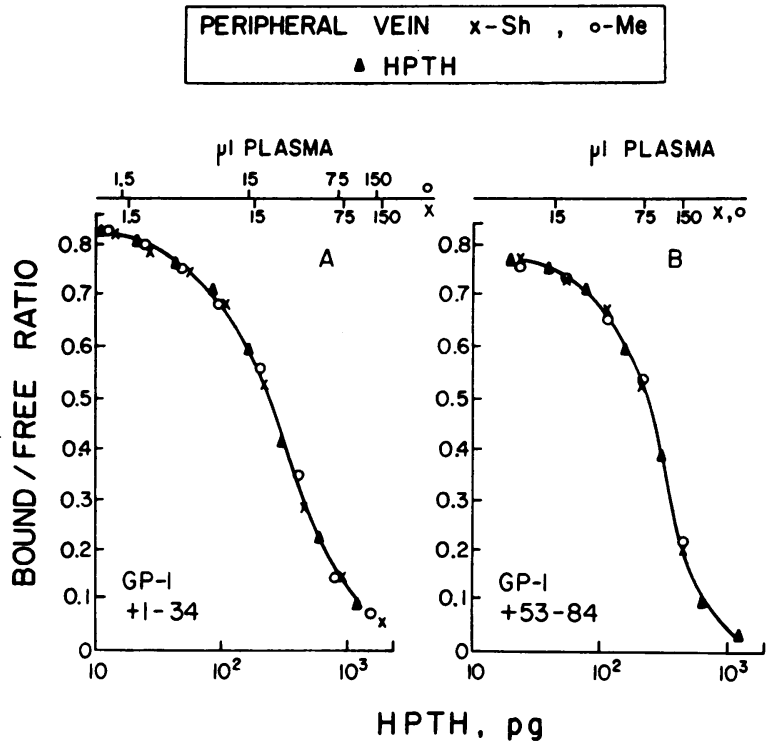

Figure 5 Immunoreactive PTH in serially diluted peripheral venous samples from patient $\mathrm{Sh}$ and $\mathrm{Me}$ in assays using, A. GP-1 $(1 / 150,000)$ preincubated with excess 1-34 and B. GP-1 $(1 / 150,000)$ preincubated with excess 53-84. Concentration is expressed in picograms of $\mathrm{HPTH}$.

mone in the circulation, rather than cleavage. could account for these observations.

\section{DISCUSSION}

In their recent review, Berson and Yalow (23) have discussed many aspects of the immunologic specificity of peptide hormones, re-emphasizing that antisera to a given hormone contain antibodies directed to different antigenic determinants and that a single antiserum may have several distinct antibody populations directed to more than one antigenic site. This has been clearly demonstrated by Aubert and Felber (24) in studies of the inhibition of binding of labeled ACTH to several antisera by a variety of synthetic fragments. Our studies of four anti-BPTH antisera demonstrate similar heterogeneity among antisera to BPTH. An antiserum can contain antibody populations with high, but varying, affinities to multiple antigenic determinants within the sequence and antisera from different animals differ in their specificity and reactivity for the determinants they recognize.

By preincubation of antisera with excess concentrations of selected fragments, we have developed antisera which recognize defined regions of the hormonal molecule. Use of these highly specific reagents has permitted studies that have not been possible with antisera of undetermined specificity.

The lower ratio of cross-reactivity of HPTH to BPTH when using GP-1 and GP-133 preincubated with excess 


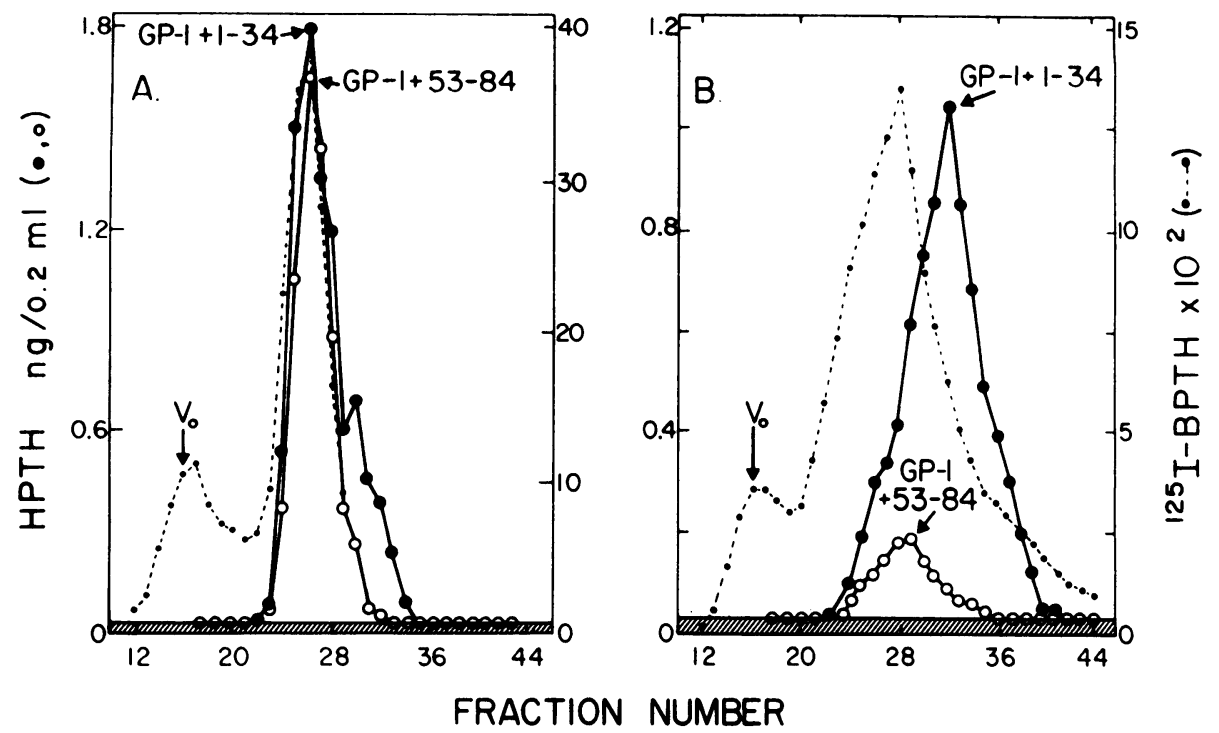

Figuke 6 Comparison of immunoreactivity in fractions after gel filtration of plasma sample: on Bio-Gel P-10, A. parathyroid effluent vein; B. peripheral vein, in assays using GP-1 $(1 / 150,000)$ preincubated with excess $1-34(\bullet-\bullet)$ and GP-1 $(1 / 150,000)$ preincubated with excess 53-84 (O- $\mathrm{O})$. Concentration is expressed in nanograms of $\mathrm{HPTH} / 0.2 \mathrm{ml}$ fraction sample. BPTH- ${ }^{125} \mathrm{I}$ was cochromatographed as marker $(\bullet-. \cdot)$. Vo marks the voill volume. The cross-hatched area represents the sensitivity limits of the radioimmunoassays.

53-84 fragment, than when these antisera are preincubated with excess 1-34, indicates that greater immunochemical similarities exist in the amino, than the carboxy, portions of the hormones. Since at least the first 21 amino-terminal residues are required for biological activity (25), these observations may mean that the immunochemical similarities are due to a greater degree of chemical homology between limited amino portions of the two hormones and that this, in turn, reflects the fact that, in all probability, certain amino-terminal regions cannot be markedly altered without loss of biological activity. Clearly, however, the significance of these immunochemical observations must await more information concerning the sequence of the human hormone, the structural requirements for biological activity and the amino acid substitutions which can be tolerated without loss of biological activity.

These observations may explain the finding that although four to five times more HPTH than BPTH is usually required for equivalent inhibition of tracer binding to GP-1 and GP-133, this ratio may vary from 2 to $8(11)$. The ratio of cross-reactivity of HPTH to BPTH depends on inhibition of tracer binding to antibody populations which recognize multiple determinants with varying reactivities. Therefore, alterations of tracer during labeling which influence the reactivity of certain antigenic determinants of tracer more than others, will have different effects on the slopes of inhibition of tracer binding by the two hormones. For example, if the amino-terminal recognition site of GP-1 is less reactive with a given tracer, the amount of HPTH required for equivalent inhibition of tracer binding will increase, relative to BPTH (Fig. 3). This indicates the inherent inadequacies of the heterologous: assay system and re-emphasizes the need to standardize assays against homologous plasma standard, as has usually been done $(26-29)$. or against homologous extracted hormone $(21,22)$.

In addition to improved specificity, assays using preincubated antisera show improved sensitivity. Invariably, lower concentrations of $\mathrm{BPTH}$ are required to inhibit tracer binding to GP-1 preincubated with excess 1-34 than to GP-1 before preincubation. Similarly, HPTH more effectively inhibits tracer binding to GP-133 preincubated with excess 53-84 than to GP-133 prior to preincubation. In both instances sensitivity is usually improved by about $20 \%$ (Fig. 3). This demonstrates that preincubation of antisera can select higher affinity antibodies from a population which is heterologous with respect to specificity and affinity, and is of particular interest because it confirms the theoretical predictions of Ekins, Newman, and O'Riordan (30).

The limitations of the radioimmunoassay, employing antisera of undetermined specificity, become apparent when applied to the study of immunoreactive PTH in man. Antisera are usually judged acceptable to measure concentrations of endogenous hormone if measurements can be made with adequate sensitivity and if slopes of 
inhibition of tracer binding by increasing aliquots of standard and sample are identical. Studies with GP-133 prove, however, that in the radioimmunoassay for $\mathrm{PTH}$, these criteria can be met by peripheral plasma PTH which consists predominantly of a hormonal fragment lacking a major, critical portion of the sequence of the intact peptide. Clearly, the nature and significance of PTH metabolism can be better understood by use of defined antisera.

Since the antibody populations of GP-1 principally recognize two widely separate major antigenic determinants, preincubation with selected fragments allows separate analysis of the concentration of each determinant. When applied to the assay of samples from the peripheral circulation of patients with hyperparathyroidism, these assays show that PTH in the circulation lacks an amino portion of its sequence, containing all or some of the 14-19 region. Since PTH in parathyroidvenous effluent and in extracts of human adenomas give identical slopes of inhibition of tracer binding, the immunochemical heterogeneity of PTH in the circulation of man observed with GP-1 can be attributed to the deficiency of the determinant requiring this region. By using the preincubated modifications of GP-1 in assays of the fractions after gel filtration of plasma samples, these observations are clarified. Whereas, there is equivalent immunoreactivity of the material coeluting with BPTH marker using the two modification of GP-1, the late eluting peak is completely devoid of aminoterminal reactivity. Since concentrations of immunoreactivity are equal when assayed with unblocked GP133, GP-133 preincubated with excess $1-34$ and $53-84$ and GP-1 preincubated with excess $1-34$, the immunoreactivity of this large fragment is probably not altered by the cleavage step. Thus, our calculations which show that less than $5-25 \%$ of the immunoreactivity in general circulation is due to intact hormone are probably accurate. The high concentration of the large fragment compared with that of intact hormone indicates that its metabolic clearance rate must be considerably slower than that for intact hormone. These observations suggest that some of the conflicting reports concerning concentrations of PTH in circulation (21, 26-29) may relate to the use of antisera which are heterologous with respect to specificity and reactivity with the multiple determinants of the bovine hormone.

Furthermore, since this large fragment contains the major antigenic determinant recognized by GP-133 which requires all or some of the 19-34 region, but lacks the determinant recognized by GP-1 which requires all or some of the 14-19 region, the location of the cleavage site can be delimited immunochemically. Cleavage must occur at a site which is carboxy-terminal to position 14 and amino-terminal to position 34 .
Since this large fragment lacks a portion of the sequence of intact hormone known to be necessary for biological activity (25), one can conclude that it cannot be biologically active. Therefore, less than 5-25\% of immunoreactive $\mathrm{PTH}$ in the general circulation of man, as detected by our antisera, is due to biologically active peptide. In addition, since all antisera thus far studied have been shown to have antibody populations which recognize multiple determinants, it seems most likely that an unblocked antiserum to native hormone which gives identical displacement slopes in assays for HPTH and samples from the peripheral circulation, must have antibodies which are sensitive to determinants, all of which are present in the large hormonal fragment. Therefore, such an antisera could not distinguish between biologically active, intact hormone and the biologically inactive, large fragment which is the dominant form of the hormone in the circulation.

Thus far, no immunoreactivity, other than for that present in intact hormone, has been identified in the assay of gel filtration fractions with GP-1 preincubated with excess 53-84. Inability to detect an amino-terminal fragment may be due to complete destruction of this sequence at the time of cleavage, or this fragment may re-enter the circulation, but be rapidly cleared. Lack of detection of this fragment, however, does not necessarily indicate its absence from the circulation. Cleavage may occur sufficiently near the antigenic determinant in the 14-19 region to lower the immunoreactivity of this fragment and thereby, increase the concentration needed for detection in assays with GP-1 preabsorbed with excess 53-84, or cleavage may occur, possibly, through this antigenic determinant, thereby destroying its immunoreactivity in this assay system.

It is impossible, at present, to predict whether this amino-terminal fragment could contain the structural requirements for biological activity.

Resolution of these problems must await the development of antisera with antibodies reactive with other amino-terminal determinants, such as those in the 1-13 region, which will permit further studies for the presence of this fragment, more precise localization of the cleavage site by applying antisera with more accurately characterized recognition sites, and more detailed analysis of the structural requirements for biological activity.

\section{ACKNOWLEDGMENTS}

The authors thank Dr. H. T. Keutmann for performing amino acid analyses and for providing hormones and hormonal fragment 53-84, and Mr. P. Dee and Mrs. C. Benney for technical assistance.

This work was supported in part by a grant from the John A. Hartford Foundation, Inc., grants AM 05205, AM 11794, and AM 04501 from the National Institutes of Health, 
and contract NAS-9-11011 from the National Aeronautics and Space Administration.

\section{REFERENCES}

1. Berson, S. A., and R. S. Yalow. 1968. Immunochemical heterogeneity of parathyroid hormone in plasma. $J$. Clin. Endocrinol. Metab. 28: 1037.

2. Canterbury, J. M., and E. Reiss. 1971. Fractionation of circulating parathyroid hormone $(\mathrm{PTH})$ in man. J. Iab. Clin. Med. 78: 814. (Abstr.)

3. Habener, J. F., D. Powell, T. M. Murray, G. P. Mayer, and J. T. Potts, Jr. 1971. Parathyroid hormone: secretion and metabolism in ition. Proc. Natl. Acad. Sci. U.S. A. 68: 2986.

4. Sherwood, L. M., J. S. Rodman, and W. B. Lundberg. 1970. Evidence for a precursor to circulating parathyroid hormone. Proc. Natl. Acad. Sci. U. S. A. 67: 1631.

5. Arnaud, C. D., G. W Sizemore, S B. Oldham, J. A. Fischer, H. S. Tsao, and E. T. Littledike. 1971. Human parathyroid hormone: glandular and secreted moleculai species. Am. J. Med. 50: 630 .

6. Martin, T. J., P. B. Greenberg, and R. A. Melick. 1972. Nature of human parathyroid hormone secreted by monolayer cell cultures. J. Clin. Endocrinol. Metab. $34: 437$.

7. Niall, H. D., H. Keutmann, R. Sauer, M. Hogan, B. Dawson, G. Aurbach, and J. T. Potts, Jr. 1970. The amino acid sequence of bovine parathyroid hormone I. Z. Physiol. Chem. (Hoppe-Seyler's). 351: 1586.

8. Brewer, H. B., Jr., and R. Ronan. 1970. Bovine parathyroid hormone: amino acid sequence. Proc. Natl. Acad. Sci. U. S. A. 67: 1862.

9. Potts, J. T., Jr., G. W. Tregear, H. T. Keutmann, H. D. Niall, R. Sauer, L. J. Deftos, B. F. Dawson, M. L. Hogan, and G. D. Aurbach. 1971. Synthesis of a biologically active $\mathrm{N}$-terminal tetratriacontapeptide of parathyroid hormone. Proc. Natl. Acad. Sci. U.S. A. 68: 63 .

10. Keutmann, H. T., G. D. Aurbach, B. F. Dawson, H. D. Niall, L. J. Deftos, and J. T. Potts, Jr. 1971. Isolation and characterization of the bovine parathyroid isohormones. Biochemistry. 10: 2779.

11. O'Riordan, J. L. H., G. D. Aurbach, and J. T. Potts, Jr. 1969. Immunological reactivity of purified human parathyroid hormone. Proc. Natl. Acad. Sci. U. S. A. $63: 692$.

12. O'Riordan, J. L. H., J. T. Potts, Jr., and G. D. Aurbach. 1971. Isolation of human parathyroid hormone. Endocrinology. 89 : 234.

13. Hunter, W. M., and F. C. Greenwood. 1962. Preparation of iodine-131 labelled human growth hormone of high specific activity. Nature (Lond.). 194: 495.

14. Marcus, R., and G. D. Aurbach. 1969. Bioassay of parathyroid hormone in vitro with a stable preparation of adenyl cyclase from rat kidney. Endocrinology. 85: 801.

15. Potts, J. T., Jr., L. M. Sherwood, J. L. H. O'Riordan, and G. D. Aurbach 1967 Radioimmunoassay of polypeptide hormones Adv. Intern. Med. 13: 183.

16. Berson, S. A., R. S. Yalow, G. D. Aurbach, and J. T.
Potts, Jr. 1963. Immunoassay of bovine and human parathyroid hormone. Proc. Natl. Acad. Sci. U. S. A. 49: 613.

17. Hales, C. N., and P. J. Randle. 1963. Immunoassay of insulin with insulin-antibody precipitate. Biochem. $I$. $88: 137$.

18. Sherwood, L. M., J. T. Potts, Jr., A. D. Care, G. P. Mayer, and G. D. Aurbach. 1966. Evaluation by radioimmunoassay of factors controlling the secretion of parathyroid hormone. Intravenous infusions of calcium and ethylenediamine tetraacetic acid in the cow and goat. Vature (Lond.). 209: 52.

19. Herbert, V., K. S. Lau, C. W. Gottlieb, and S. J. Bleicher. 1965. Coated charcoal immunoassay of insulin. J. Clin. Endocrinol. Metab. 25: 1375.

20. Yalow, R. S., and S. A. Berson. 1968. General principles of radioimmunoassay. U. S. A. E. C. Symp. Ser. 13. 7.

21. Potts, J. T., Jr., T. M. Murray, M. Peacock, H. D. Niall, G. W. Tregear, H. T. Keutmann, D. Powell, and L. J. Deftos. 1971. Parathyroid hormone: sequence, synthesis, immunoassay studies. Am. J. Med. 50: 639 .

22. Powell, D., P. M. Shimkin, J. L. Doppman, S. Wells, G. D. Aurbach, S. J. Marx, A. S. Ketcham, and J. T. Potts, Jr. 1972. Primary hyperparathyroidism. Preoperative tumor localization and differentiation between adenoma and hyperplasia. N. Engl. J. Med. 286: 1169.

23. Berson, S. A., and R. S. Yalow. 1971. Immunologic specificity of peptide hormones. In Structure-Activity Relationships of Protein and Polypeptide Hormones. M. Margoulies, and F. C. Greenwood, editors. International Congress Series, No. 241. Excerpta Medica Foundation, Publishers. Amsterdam. 38.

24. Aubert, M. L., and J. P. Felber. 1969. Studies on ACTH-binding antibodies: characterization of immunological specificities. Acta Endocrinol. 62: 521.

25. Aurbach, G. D., H. T. Keutmann, H. D. Niall, G. W. Tregear, J. L. H. O’Riordan, R. Marcus, S. J. Marx. and J. T. Potts, Jr. 1971. Structure, synthesis and mechanism of action of parathyroid hormone. Recent Prog. Horm. Res. 28: 353.

25. Berson, S. A., and R. S. Yalow. 1966. Parathyroid hormone in plasma in adenomatous hyperparathyroidism, uremia and bronchogenic carcinoma. Science (Wash. D. C.). $154: 907$.

27. Reiss, E., and J. M. Canterbury. 1968. A radioimmunoassay for parathyroid hormone in man. Proc. Soc. Exp. Biol. Med. 128 : 501.

28. Arnaud, C. D., H. S. Tsao, and E. T. Littledike. 1971. Radioimmunoassay of human parathyroid hormone in serum. J. Clin. Invest. 50:21.

29. Reiss, E., and J. M. Canterbury. 1969. Primary hyperparathyroidism. Application of radioimmunoassay to dif ferentiation of adenoma and hyperplasia and to preoperative localization of hyperfunctioning parathyroid glands. N. Engl. J. Med. 280: 1381.

30. Ekins, R. P., G. B. Newman, and J. L. H. O'Riordan. 1968. Theoretical aspects of "saturation" and radioimmunoassay. U.S. A. E. C. Symp. Ser. 13. 59. 